\title{
Magnetic Birefringence Study of the Magnetic Core Structure of Ferritin
}

\author{
M. Koralewski ${ }^{a, *}$, M. Pochylski ${ }^{a}$, Z. Mitróová ${ }^{b}$, L. Melníková ${ }^{b}$, J. Kováč ${ }^{b}$, \\ M. TIMKO ${ }^{b}$ AND P. KOPČANSKÝ ${ }^{b}$ \\ ${ }^{a}$ Faculty of Physics, Adam Mickiewicz University, Umultowska 85, 61-614 Poznań, Poland \\ ${ }^{b}$ Institute of Experimental Physics, SAS, Watsonova 47, 04001 Kosice, Slovakia
}

\begin{abstract}
Magnetically induced optical birefringence $(\Delta n)$ was measured for magnetoferritin and horse spleen ferritin aqueous suspensions. The $\Delta n$ for magnetoferritin was described in the frame of the Langevin formalism taking into account distribution of core diameter. The established average magnetic dipole moment and core diameter is equal to about $460 \mu_{\mathrm{B}}$ and $3 \mathrm{~nm}$, respectively. It was shown that magnetic birefringence and the Cotton-Mouton constant can be powerful parameters in identification of the magnetic core structure of ferritin, especially useful in biomedicine.
\end{abstract}

PACS: 78.20.Ls, 87.15.-v, 87.85.jf

\section{Introduction}

The ferritin is a protein which serves as a storage capsule of iron inside living organisms. The protein itself (apoferritin) is an almost perfect sphere, with outer diameter about $12 \mathrm{~nm}$, hollow inside, with about 7 to $8 \mathrm{~nm}$ inner diameter. Recently it was shown that the hollow structure of apoferritin can be exploited as an excellent reaction vessel for nanoscale synthesis of different minerals. Up to date many compounds have been synthesised within this protein cage $[1,2]$ and potential applications in electronics and biomedicine were discussed [3-5]. The ferritin-mineral complex with a magnetite (or a maghemite) as a core mineral, is called magnetoferritin (MFer) [1]. Discovery of biological magnetite in the human brain [6] and relation of its presence with neurodegenerative disorders [7] have prompted investigation of physicochemical properties of ferritin and magnetoferritin $[8,9]$. Of particular interest is the search for methods allowing detection of magnetite inside ferritin proteins both in vitro and in vivo.

The static birefringence of dilute ferrofluids exposed to external magnetic field results from the orientation of optically anisotropic particles along the magnetic field direction. The degree of orientation depends on the energy related with the interaction between particle's magnetic moments (both induced and/or permanent) and the magnetic field. Thus magnetic field induced birefringence $(\Delta n)$ can be the effect potentially able to be used for discrimination between magnetic core structure of ferritin and magnetoferritin which is the subject of present study.

\section{Experiment and calculations}

A natural biogenic ferritin was the horse spleen ferritin (HSF) (lot 079K7 001) obtained from Sigma-Aldrich (iron concentration $c^{\mathrm{Fe}}=7.1 \mathrm{~g} / \mathrm{L}$ ) and used without further treatment. Synthetic ferritin, i.e., MFer was de-

\footnotetext{
* corresponding author; e-mail: koral@amu.edu.pl
}

rived from equine spleen apoferritin (Sigma-Aldrich) using the synthesis scheme described in our papers $\left(c^{\mathrm{Fe}}=\right.$ $0.126 \mathrm{~g} / \mathrm{L}$ ) [10, 11]. The real loading factor (LF), i.e. number of iron ions inside ferritin cage, estimated using a UV-Vis spectrometry, was found to be $L F=1250$. The superparamagnetic properties of the synthesised MFer were tested and the blocking temperature $T_{\mathrm{B}}$ was established to be $\approx 25 \mathrm{~K}$, details are described in our papers $[10,11]$.

Optical birefringence was measured using the laboratory made setup described elsewhere [12], which allows precise measurements of the angle of rotation of the polarization plane, $\Theta$ for light passing through the sample placed in a magnetic field in the Voigt configuration. The light beam from a He-Ne laser $(\lambda=632.8 \mathrm{~nm})$ was used.

Taking into account permanent magnetic moments of a mineral core, the orientational effect can be described in terms of the Langevin formalism. The value of birefringence can be calculated using the relation [13-16]:

$$
\Delta n=\Delta n_{\mathrm{s}} L_{2}(\xi),
$$

where $\Delta n_{\mathrm{s}}$ is the saturation birefringence, also for simplicity we assume that anisotropy parameter $\sigma[14]$ gives a contribution to Eq. (1) equal to 1. The second order Langevin term $L_{2}(\xi)=1-3 L(\xi) / \xi, L(\xi)$ is the first order or usual Langevin function, $\xi$ is a Langevin parameter i.e. $\xi=\mu_{\mathrm{m}} H / k_{\mathrm{B}} \mathrm{T}$, where $\mu_{\mathrm{m}}$ relates to nanoparticles magnetic moment, $H$ is magnetic field strength, $k_{\mathrm{B}}$ is Boltzmann constant, and $T$ is temperature.

The value of $\mu_{\mathrm{m}}$ can be directly obtained from the fit of Eq. (1) to experimental static birefringence measured as a function of $H$. If the spherical shape of the particles can be assumed, then the value of magnetic moment is related to the particles diameter $D$ and its saturation magnetization $m_{\mathrm{s}}$ through the relation

$$
\mu_{\mathrm{m}}=m_{\mathrm{s}} \pi D^{3} / 6 \text {. }
$$

As follows from Eq. (2), if $m_{\mathrm{s}}$ is known from independent measurements, the fitting procedure allows us to estimate the particle diameter $D$ (and vice versa).

When the system is a polydisperse suspension, then Eq. (1) needs to be rewritten taking into account the 
particle size distribution

$$
\Delta n=\Delta n_{\mathrm{s}} \frac{\int L_{2}(\xi) P(D) D^{3} \mathrm{~d} D}{\int P(D) D^{3} \mathrm{~d} D},
$$

where $P(D)$ is the most commonly used log-normal size distribution function with $D_{0}$ and $s$ as parameters (see Table) [10, 17]. The particle shape and their distribution are not taken in explicit form into account in the above model, however particle shape contributes to $\Delta n_{\mathrm{s}}$ value.

TABLE

The values of parameters obtained in fitting procedure i.e. $\Delta n_{\mathrm{s}}, D_{0}, s$, with the value of $\chi^{2}$ describing the goodness of the fit; together with average magnetic moment $\left\langle\mu_{\mathrm{m}}\right\rangle$, diameter $\langle D\rangle$ and their standard deviation $\beta$ for various values of core magnetisation.

\begin{tabular}{c|c|c|c}
\hline \hline $\begin{array}{c}\text { Magnetisation } \\
{[\mathrm{kA} / \mathrm{m}]}\end{array}$ & 323.7 & 356.9 & 518 \\
\hline$\Delta n_{\mathrm{s}} \times 10^{6 a}$ & 2.021 & 2.018 & 2.012 \\
$D_{0}[\mathrm{~nm}]$ & 2.55 & 2.48 & 2.21 \\
$s$ & 0.527 & 0.526 & 0.523 \\
$\langle D\rangle[\mathrm{nm}]$ & 2.92 & 2.84 & 2.54 \\
$\beta[\mathrm{nm}]$ & 1.65 & 1.60 & 1.42 \\
$\left\langle\mu_{\mathrm{m}}\right\rangle\left[\mu_{\mathrm{B}}\right]$ & 457 & 463 & 479 \\
$\chi^{2}$ & 0.0629 & 0.0631 & 0.0636 \\
\hline
\end{tabular}

${ }^{a}$ At RT and $\lambda=632.8 \mathrm{~nm}$, for $c^{\mathrm{Fe}}=1 \mathrm{~g} / \mathrm{L}$.

For the low magnetic field region, i.e., when the $\xi \ll 1$, the Langevin function can be approximated by $L_{2}(\xi) \cong \xi^{2} / 15$. In this limit the birefringence is proportional to the square of magnetic field. The proportionality constant is known as the Cotton-Mouton constant, $C^{\mathrm{CM}}[18]$ :

$$
\Delta n=C^{\mathrm{CM}} \lambda H^{2} \text {. }
$$

\section{Results and discussion}

Magnetically induced optical birefringence $\Delta n$ was measured for HSF aqueous suspension and is shown in Fig. 1. It was found that the specific magnetic birefringence (i.e. $\Delta n$ divided by the iron concentration $c^{\mathrm{Fe}}$ in $\mathrm{g} / \mathrm{L}$ ) is concentration independent [10]. Such results suggest that no aggregation of HSF appears. In the whole magnetic field range, i.e., up to $20 \mathrm{kOe}$ no indication of $\Delta n$ saturation was noticed and a linear relation between $\Delta n$ and $\mathrm{H}^{2}$ is fulfilled (see inset of Fig. 1). This means that we are in a low-magnetic-field limit and only the Cotton-Mouton constant, $C^{\mathrm{CM}}$, can be calculated. The specific $\mathrm{CM}$ constant values (i.e. $C^{\mathrm{CM}} / c^{\mathrm{Fe}}$ ) was found to be $C^{\mathrm{CM}}=6.97 \times 10^{-14} \mathrm{~m} \mathrm{~A}^{-2}$, which is in a good agreement with previously published results [19].

Distinctly different behavior of magnetic birefringence can be observed for MFer (Fig. 2). The $\Delta n(H)$ shows clearly non-quadratic dependence. The $\Delta n$ value raises very sharply with increasing magnetic field, and reaches a value close to full saturation at the highest field obtainable in the experiment, i.e. $20 \mathrm{kOe}$. For low magnetic fields $(H<200 \mathrm{Oe}) \Delta n$ is proportional to $H^{2}$, which allows the calculation $C^{\mathrm{CM}}$ using Eq. (4). Low

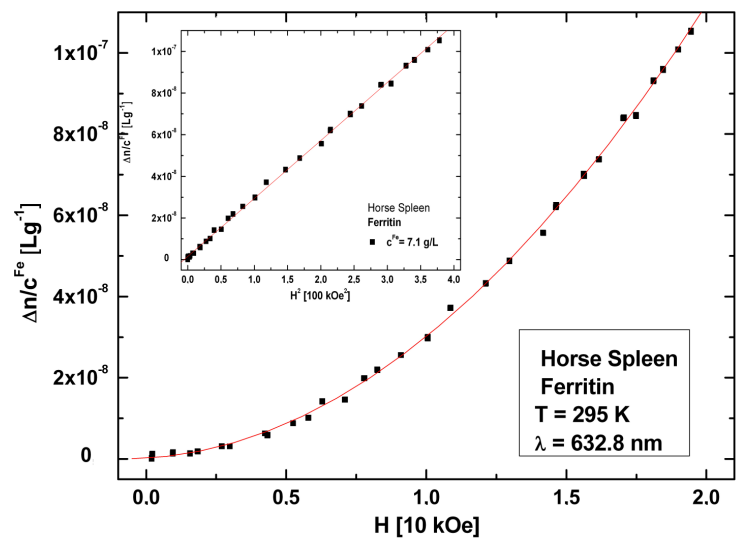

Fig. 1. Reduced magnetic birefringence $\Delta n / c^{\mathrm{Fe}}$ as a function of the applied magnetic field $H$ for HSF aqueous suspension $\left(c^{\mathrm{Fe}}=7.1 \mathrm{~g} / \mathrm{L}\right)$. The inset shows a plot of $\Delta n / c^{\mathrm{Fe}}$ vs. $H^{2}$. The solid line is a linear fit to experimental points to obtain the Cotton-Mouton constant.

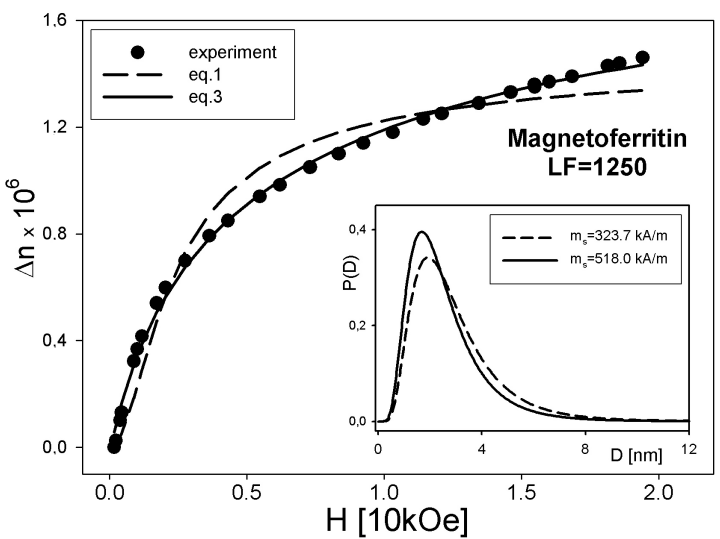

Fig. 2. The reduced magnetic birefringence $\Delta n / c^{\mathrm{Fe}}$ as a function of the applied magnetic field for MFer aqueous suspension $\left(c^{\mathrm{Fe}}=0.126 \mathrm{~g} / \mathrm{L}\right)$. The dashed and solid line are the best fit by a Langevin function using Eqs. (1) and (3), respectively. The inset shows the particle size distribution function obtained for two magnetisation values.

field region for MFer is at least two orders of magnitude smaller than for HSF. This means that the $C^{\mathrm{CM}}$ for the former compound will be more than four orders higher. The specific CM constant for MFer was found to be $C^{\mathrm{CM}}=3.45 \times 10^{-10} \mathrm{~m} \mathrm{~A}^{-2}$.

Magnetic birefringence dependence for MFer (Fig. 2) can be interpreted in terms of the Langevin formalism. The best fit to the experimental data using Eq. (1) (for discrete value of particle diameter, i.e., monodisperse ferrofluids) is obtained for the average magnetic moment of the particle $\mu_{\mathrm{m}}=8501 \mu_{\mathrm{B}}$. Figure 2 presents very poor agreement between experimental data and the universal Langevin curve $\left(\chi^{2}=0.636\right)$. This value $\mu_{\mathrm{m}}$ gives us average magnetic moment per Fe ion in MFer cage equal to $6.8 \mu_{\mathrm{B}}$. The obtained value is much too high if we compare it to the value obtained for the nanoscale magnetite (i.e. $0.9 \mu_{\mathrm{B}}$ or $0.83 \mu_{\mathrm{B}}$ ) [10] or bulk magnetite $\left(1.33 \mu_{\mathrm{B}}\right)$. 


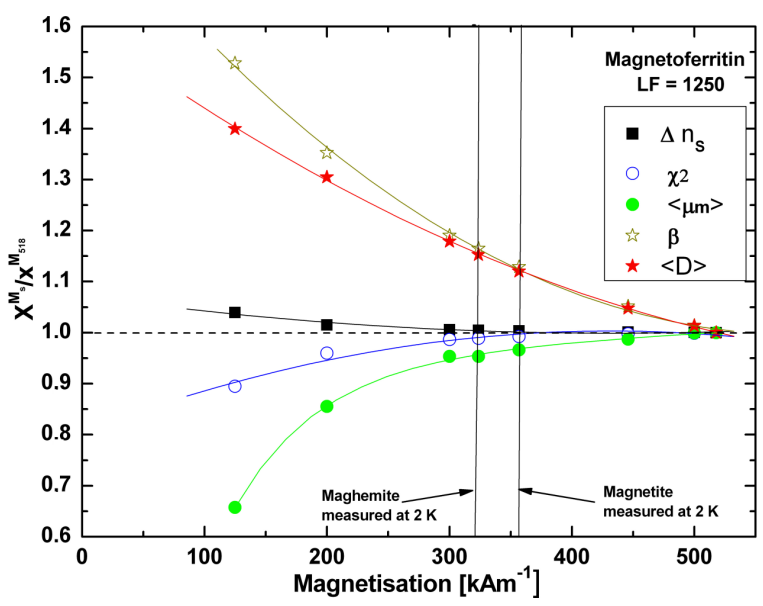

Fig. 3. Normalized coefficients dependence on saturation magnetisation, $m_{\mathrm{s}}$. The coefficients were obtained in fitting procedure using Eq. (3) (see Table).

It is even higher than the theoretical value for the single iron ion in high spin state $\left(5.9 \mu_{\mathrm{B}}\right)$. This observation, together with poor fit, suggests that the model expressed by Eq. (1) is too simple. The simple development is to assume a distribution of MFer magnetic core size (Eq. (3)) instead of uni-modal proposition (Eq. (1)). It should be noted that the model expressed by Eq. (3) has four parameters, while Eq. (1) has only two free fitting parameters. Equation (2) makes the saturation magnetization, $m_{\mathrm{s}}$, and the particle diameter, $D$, strongly dependent. For this reason, $m_{\mathrm{s}}$, of the particle's material should be known and kept fixed during fitting procedure. We can assume that whole MFer core material is made of maghemite or magnetite, for which $m_{\mathrm{s}}$ values, measured at $2 \mathrm{~K}$, is equal to $323.7 \mathrm{kA} / \mathrm{m}$ or $356.9 \mathrm{kA} / \mathrm{m}$, respectively. With the above assumptions, the best fit of Eq. (3) to the experimental data appears much better than observed when using Eq. (1). As it is not easy to decide whether the crystal structure has a core, it was tested how the average magnetic moment and core diameter depend on the value of magnetisation used in fitting procedure.

The fitting results are summarized in Table and shown in Fig. 3 as normalized parameters i.e. parameter at given value of $m_{\mathrm{s}}$ was divided by the parameter obtained for maximal $m_{\mathrm{s}}=518 \mathrm{kA} / \mathrm{m}$. It may be noticed that for magnetisation in the range 300 to $518 \mathrm{kA} / \mathrm{m}$ the average magnetic moment per particle/core $\mu_{\mathrm{m}}$ does not change much and the goodness of the fit as measured by $\chi^{2}$ is comparable (see Fig. 3). Much higher changes for diameters and especially their standard deviation may be noticed. Above result suggests that up to certain LF the core of magnetoferritin will show rather high polydispersity although it is placed in very monodisperse cage of apoprotein.

\section{Conclusion}

The magnetic birefringence of biogenic (HSF) and synthetic (MFer) ferritin was studied. The established average magnetic dipole moment and core diameter is equal to about $460 \mu_{\mathrm{B}}$ and $3 \mathrm{~nm}$, respectively. The CottonMouton constants are roughly four orders of magnitude different for both studied compounds. Direct observation of saturation effect for MFer, together with the lack of such effect in HSF, should allow for discrimination of the core of unknown ferritin. It would be highly recommended to use described magnetooptical method to identify magnetic structure of brain ferritin.

\section{Acknowledgments}

M.K. acknowledges Prof. R. Wojciechowski (AMU) for fruitful discussions. The work was supported by the Ministry of Science and Higher Education, grant No. NN202 124535 as well as the project No. 26000120033 and No. 26220120033 in the frame of Structural Funds of European Union, Centre of Excellence of SAS Nanofluid and VEGA 0077.

\section{References}

[1] F.C. Meldrum, B.R. Heywood, S. Mann, Science 257, 522 (1992).

[2] M. Uchida, S. Kang, C. Reichardt, K. Harlen, T. Duglas, Biochim. Biophys. Acta 1800, 834 (2010).

[3] M.T. Klem, J. Mosolf, M. Young, T. Douglas, Inorg. Chem. 47, 2237 (2008).

[4] I. Yamashita, K. Iwahori, S. Kumagai, Biochim. Biophys. Acta 1800, 846 (2010).

[5] K. Ohara, Y. Uraoka, T. Fuyuki, I. Yamashita, T. Yaegashi, M. Moniwa, M. Yoshimaru, Jpn. J. Appl. Phys. 48, 04C153 (2009).

[6] J.L. Kirschvink, A. Kobayashi-Kirschvink, B.J. Woodford, Proc. Natl. Acad. Sci. USA $\mathbf{8 9}$, 7683 (1992).

[7] C. Quintana, Mini-Rev. Med. Chem. 7, 961 (2007).

[8] V.C. Jordan, M.R. Caplan, K.M. Bennett, Magn. Res. Med. 64, 1260 (2010).

[9] A. Figuerola, R. Di Corato, L. Manna, T. Pellegrino, Pharmacol. Res. 62, 126 (2010).

[10] M. Koralewski, M. Pochylski, Z. Mitróová, M. Timko, P. Kopčanský, L. Melníková, J. Magn. Magn. Mater. 323, 2413 (2011).

[11] Z. Mitróová, L. Melníková, J. Kováč, M. Timko, P. Kopčanský, Acta Phys. Pol. A 121, 1318 (2012).

[12] M. Koralewski, M. Pochylski, J. Gierszewski, J. Magn. Magn. Mater. 323, 1140 (2011).

[13] C.T. O'Konski, T. Yoshioka, W.H. Orttung, J. Phys. Chem. 63, 1558 (1959).

[14] E. Hassamony, E. Dubois, J.-C. Bacri, R. Perzynski, Yu.L. Raikher, V.I. Stepanov, Eur. Phys. J. B 5, 859 (1989).

[15] P.C. Scholten, IEEE Trans. Magn. 11, 1400 (1975).

[16] U. Neitzel, K. Barner, Phys. Lett. A 63, 327 (1977).

[17] J. Popplewell, L. Sakhnini, J. Magn. Magn. Mater. 149, 72 (1995).

[18] H. Mouton, Int. Crit. Tables 7, 109 (1934).

[19] M. Pankowska, A. Dobek, J. Chem. Phys. 131, 015105 (2009). 